\title{
Identification of the $c s p$ gene and molecular modelling of the CspA-like protein from Antarctic soil-dwelling psychrotrophic bacterium Psychrobacter sp. B6
}

\author{
Agnieszka Kaufman-Szymczyk ${ }^{1 \star}$, Arkadiusz Wojtasik ${ }^{2}$, Paweł Parniewski², \\ Aneta Białkowska1 ${ }^{1}$ Karolina L. Tkaczuk ${ }^{1}$ and Marianna Turkiewicz ${ }^{1}$ \\ ${ }^{1}$ Institute of Technical Biochemistry, Technical University of Lodz, Eódź, Poland; ${ }^{2}$ Centre of Medical Biology, \\ Polish Academy of Sciences, Łódź, Poland
}

Received: 04 August, 2008; revised: 12 January, 2009; accepted: 16 February, 2009 available on-line: 24 February, 2009

\begin{abstract}
We cloned and sequenced the $\operatorname{cs} p A$-like gene from a psychrotrophic Antarctic soil-dwelling bacterial strain Psychrobacter sp. B6. The gene is $213 \mathrm{bp}$ long and shows $99 \%$ and $98 \%$ sequence identity with the Psychrobacter cryohalolentis K5 gene encoding a cold-shock DNA-binding domain protein and the Psychrobacter arcticus transcriptional regulator-CspA gene, respectively. The protein encoded by the Psychrobacter sp. B6 cspA-like gene shows $100 \%$ identity with the two proteins mentioned above, and also $61 \%$ sequence identity with CspB from Bacillus subtilis and Csp from Bacillus caldolyticus, and 56\% - with Escherichia coli CspA protein. A three-dimensional model of the CspA-like protein from Psychrobacter sp. B6 was generated based on three known structures of cold shock proteins: the crystal structure of the major cold shock protein from Escherichia coli (CspA), the NMR structure of the latter protein, and the NMR structure of Csp from Thermotoga maritima. The deduced structure of the CspA-like protein from Psychro-

bacter sp. B6 was found to be very similar to these known structures of Csp-like proteins.
\end{abstract}

Keywords: Cold-living bacterium, cold shock protein, CspA-like protein, RNA chaperone, nucleic acid binding

\section{INTRODUCTION}

Throughout evolution live organisms have developed many adaptations to harsh and hostile environmental conditions and the changes in these conditions. A sudden temperature downshift is one of principal environmental stresses encountered by bacteria, in particular those inhabiting the permanently cold areas of the Earth. It results in a significant decrease in cell membrane fluidity and disturbs virtually all intracellular processes, including transcription and translation (Russell et al., 1995; Jones \& Inouye, 1996). To survive under such adverse conditions, microorganisms produce a number of cold- induced proteins. A family of small proteins named cold shock proteins (Csp) is the most essential for cell survival.

The first cold shock protein CspA from Escherichia coli was identified in 1987 (Jones et al., 1987). From that time, the cold shock response has been extensively investigated. In contrast to the heat shock response it is, however, still not so well understood.

The cellular cold shock response seems to be universal (Jones \& Inouye, 1994) as it occurs in almost every microbial cell after a rapid drop of temperature and always brings about synthesis of specific proteins - the cold shock proteins. Csps

${ }^{\square}$ Corresponding author: Agnieszka Kaufman-Szymczyk, Institute of Technical Biochemistry (IBT), Technical University of Lodz, Stefanowskiego 4/10, 90-924 Łódź, Poland; phone: (48) 42631 3442; fax: (48) 42636 6618; e-mail: agakauf@yahoo. com

^Agnieszka Kaufman-Szymczyk and Arkadiusz Wojtasik contributed equally to this work

Abbreviations: Csp, cold shock protein; CSD, cold shock domain; NMR, nuclear magnetic resonant; OB-fold protein, oligonucleotide binding protein; RNP, single stranded nucleic acids binding motif; ssDNA, single-stranded DNA. 
have been found in all types of bacteria, i.e. in thermophiles, mesophiles and psychrophiles (Mueller et al., 2000; Ermolenco \& Makhatadze, 2002; Phadtare et al., 2003). Several homologous copies of csp genes have been identified in the majority of known bacterial genomes. Nine csp homologues, $\operatorname{csp} A-\operatorname{csp} I$, were found in E. coli (Yamanaka et al., 1998), five of these genes are expressed in response to a sudden drop in temperature (Nakashima et al., 1996; Wang et al., 1999; Uppal et al., 2008). Three csp genes, $\operatorname{csp} B, C$ and $D$ were found in B. subtilis (Schindler et al., 1999) and two cold shock genes, $\operatorname{csp} A$ and $\operatorname{csp} G$, both coldinduced, were identified in a deep-sea psychrophilic bacterium S. violacea (Fujii et al., 1999). Four genes encoding a small cold-induced Csp from the Antarctic Streptomyces sp. AA 8321 have also been cloned recently (Kim et al., 2007).

Csps are believed to act as regulators of transcription and translation (Gualerzi et al., 2003). They also act as molecular chaperones because they nonspecifically bind single-stranded nucleic acids and destabilize their secondary structures at low temperature (Jiang et al., 1997; Lopez \& Makhatadze, 2000; Bae et al., 2000; Phadtare et al., 2002; Zeeb \& Balbach, 2003; Zeeb et al., 2006). In general, Csps are involved in establishing a "new" cell balance, which in turn facilitates survival at low temperature.

It is postulated that the cold shock proteins also enhance microbial tolerance to freezing due to cryoprotectant properties (Willmsky et al., 1992). It was found that viability of cells of some bacterial strains increased when they had been subjected to a cold-shock prior to freezing. It implies that the coldshock invokes cryotolerance (Kim \& Dunn, 1997; Jeffreys et al., 1998; Kim et al., 1998).

Studies on cold shock proteins have focused on those produced by meso- and thermophiles. Three dimensional structures have been determined for four cold shock proteins from mesophilic, thermophilic and hyperthermophilic bacteria such as: Escherichia coli Ec-CspA (Newkirk et al., 1994; Schindelin et al., 1994; Feng et al., 1998), Bacillus subtilis BsCspB (Schuchel et al., 1993; Schindelin et al., 1993), Bacillus caldolyticus Bc-Csp (Mueller et al., 2000) and Thermotoga maritima Tm-Csp (Kremer et al., 2001). All of these small cold shock proteins have a very similar conformation and contain five anti-parallel $\beta$-strands folded into a $\beta$-barrel structure. This particular molecule fold is characteristic for the OBfold family of proteins (Ermolenko \& Makhatadze, 2002; Horn et al., 2007). Interestingly, eukaryotic cold shock domains (CSDs), located in Y-box proteins, are involved in nucleic acid binding and show relatively high sequence and structure similarity to prokaryotic Csps (Kloks et al., 2002; 2004).

The three-dimensional structure of a psychrophilic or psychrotrophic Csp has not yet been determined. We have modelled the 3D structure of the CspA-like protein from the psychrotrophic bacterium Psychrobacter sp. B6 on the basis of the sequence of its gene. This is the first model describing a Csp molecule from a cold-adapted bacterium.

\section{MATERIALS AND METHODS}

Bacterial strain and growth conditions. The psychrotrophic bacterial strain B6 was isolated from samples of Antarctic soil derived from the coast of Admiralty Bay, King George Island (close to the Henryk Arctowski Polish Antarctic Station). The strain B6, a Gram-negative coccobacillus, was identified as Psychrobacter sp. based on partial sequence of its 16S rRNA gene (deposited in GenBank/EMBL, accession number EF028072).

Shaken cultures of this strain were grown at $20^{\circ} \mathrm{C}$ and 130 r.p.m. in a liquid nutrient medium (bacto beef extract, $0.3 \%$; bacto peptone, $0.5 \%$; yeast extract, $0.25 \%$; $\mathrm{NaCl}, 0.5 \%$; glucose, $0.1 \%$ ) until the $\mathrm{OD}_{540}$ reached 0.4-0.6.

DNA isolation. Cells from 5-ml cultures were harvested by centrifugation at $12000 \times g$ at $4^{\circ} \mathrm{C}$ for $20 \mathrm{~min}$. The pellet was suspended in $200 \mu \mathrm{l} \mathrm{TE}$ buffer (10 mM Tris/HCl, pH 8.0, 1 mM EDTA) and treated with $10 \mathrm{mg} \mathrm{ml}^{-1}$ lysozyme at $37^{\circ} \mathrm{C}$ for $12 \mathrm{~h}$. Two drops of glass beads (Invitrogen) suspended in TE buffer were added and the cells were mechanically disrupted using a Braun MKS grinder for $1 \mathrm{~min}$, at maximal speed. Then the cells were incubated with DNAzol Reagent (Invitrogen) in accordance with the manufacturer's instruction. The glass beads and unbroken cells were removed by centrifugation at $12000 \times \mathrm{g}$ at $4^{\circ} \mathrm{C}$ for $10 \mathrm{~min}$. The lysate was extracted three times with phenol/chloroform/isoamyl alcohol (25:24:1, by vol.) and then DNA was precipitated with $95 \%$ ethanol and dissolved in nuclease-free water.

PCR amplification. The PCR primers Psch 8 forward: 5'-AATCGGTGATGACACACGTC-3' and Psch 8 reverse: 5'-TACTGTCATTAAGCGCGCTG-3' were designed based on flanking regions of two major Csp gene sequences from Psychrobacter cryohalolentis K5 and Psychrobacter arcticus 273-4 (NCBI Genome Database, acc. no. NC_007969 and NC_007204, respectively). The amplified PCR product was 330 bp in length. PCR amplification was performed in a T-Personal thermocycler (Biometra) using $1 \mathrm{U}$ of Taq polymerase (Invitrogen). PCR conditions were as follows: denaturation at $94^{\circ} \mathrm{C}$ for $3 \mathrm{~min}$, followed by amplification for 35 cycles at $94^{\circ} \mathrm{C}$ for $1 \mathrm{~min}, 52^{\circ} \mathrm{C}$ at $1 \mathrm{~min}$, and $72^{\circ} \mathrm{C}$ for $2 \mathrm{~min}$, followed by a final extension at $72^{\circ} \mathrm{C}$ for $8 \mathrm{~min}$. The amplified DNA fragment was purified by electrophoresis in 1.5\% agarose gel (in TAE buffer). The gels were stained with ethid- 
ium bromide $\left(1 \mu \mathrm{g} \mathrm{m}^{-1}\right)$ and analyzed in UV light (Fc8800, Alphainnotech).

Cloning of the csp-like gene from Psychrobacter sp. B6. Cloning of the PCR product harboring the csp-like gene was performed using the pGEM T-easy system (Promega) and DNA mixtures were transformed into E. coli DH5 $\alpha$. The clones were selected by the X-gal/IPTG screening method (Sambrook \& Russell, 2001). Plasmid DNA was prepared by alkaline lysis of culture using a Promega kit (Vizard Plus SV Minipreps) and five independent clones were sequenced at the DNA Sequencing and Oligonucleotide Synthesis Laboratory, Institute of Biochemistry and Biophysics, Polish Academy of Sciences (Warszawa, Poland). The nucleotide sequence of the CspA-like protein gene from Psychrobacter sp. B6 was deposited in the GenBank/EMBL nucleotide databases (accession number EF094463).

Sequence data analysis and molecular modelling. GenBank was scanned for related sequences by the BLAST algorithm. Nucleotide and amino-acid sequences were aligned by using the computer program Vector.

Protein structure prediction was carried out via the GeneSilico MetaServer (http://genesilico.pl/ meta/, Kurowski \& Bujnicki, 2003), which is a convenient gateway to a number of publicly available online services for secondary structure prediction.

The alignment between the sequences of the CspA-like protein from Psychrobacter sp. B6 and its counterparts from the database was the first step of homology modelling. A set of alternative models was generated using MODELLER (Sali \& Blundell, 1993) and SWISS-MODEL (Peitsch, 1996), and the models were scored using VERIFY3D (Luthy et al., 1992) via the COLORADO3D server (Sasin \& Bujnicki, 2004). The final model was obtained using the 'Frankenstein's monster' approach (Kosinski et al., 2003) which comprises cycles of realignment in poorly scored regions and merging the best scoring fragments. The sequence-structure fit in the final model was also evaluated using VERIFY3D (Luthy et al., 1992), while the stereochemical parameters were assessed with PROCHECK (Laskowski et al., 1993).

Modelling of the Psychrobacter sp. B6 CspAlike protein was performed using templates selected based on the results of the GeneSilico MetaServer. The templates used for the modelling were PDB ID: $1 \mathrm{mjc}$ - the crystal structure of the major cold shock protein from E. coli (CspA), (Schindelin et al., 1994), PDB ID: 3mef - the NMR structure of the same protein solved by Feng et al. (1998), and PDB ID: 1g6p - the NMR structure of Csp from Thermotoga maritima (Kremer et al., 2001). However, since the superposition of all the aforementioned structures gave a highly consistent topology, as the final template only the X-ray structure was used.

\section{RESULTS}

Cloning and sequencing of CspA-like gene from Psychrobacter sp. B6

The predicted $\operatorname{csp} A$-like ORF from Psychrobacter sp. B6 consists of $213 \mathrm{bp}$ and shows 99\% sequence identity with the gene of a DNA-binding domain protein from Psychrobacter cryohalolentis K5 and $98 \%$ with the gene of the transcriptional regulator-CspA from Psychrobacter arcticus 273-4 (Fig. 1). The predicted amino-acid sequence of the protein encoded by $\operatorname{csp} A$-like gene is identical (Fig. 2) with that of the cold-shock DNA-binding domain protein from $P$. cryohalolentis $\mathrm{K} 5$ and the transcriptional regulator-CspA from $P$. arcticus 273-4. Besides, these proteins display $56 \%$ identity with $E$. coli major cold shock protein CspA, and $61 \%$ with B. subtilis CspB and B. caldolyticus Csp (Fig. 3).

\section{Molecular modelling of CspA-like protein}

Modelling of the Psychrobacter sp. B6 CspAlike protein was based on known structures of major cold shock proteins from E. coli (CspA), (Schin-

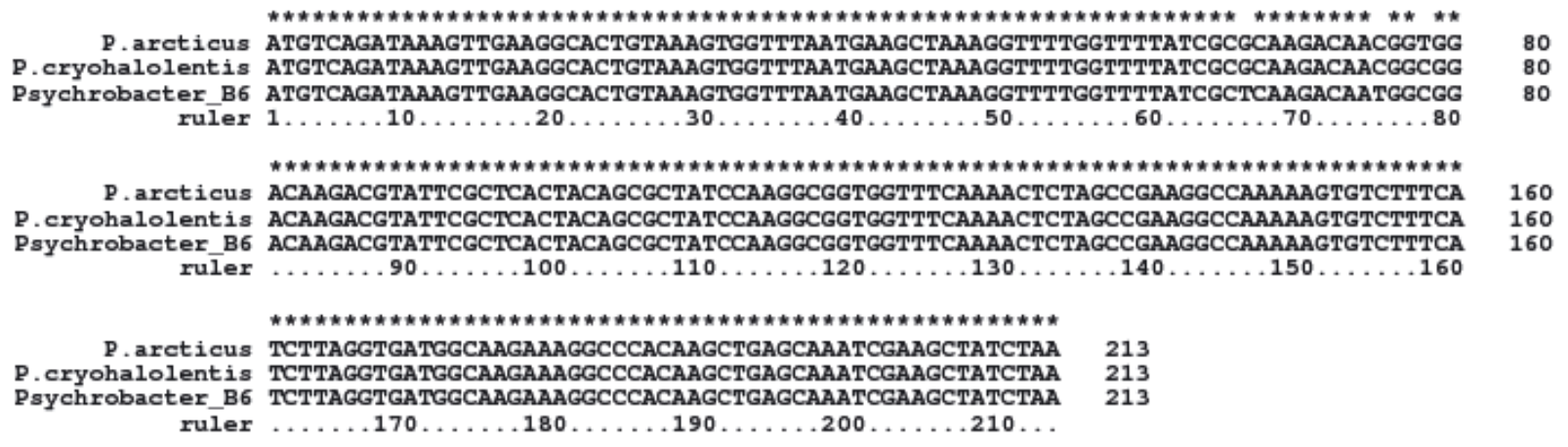

Figure 1. Sequence alignment of genes encoding cold shock proteins from Psychrobacter genus.

Psychrobacter arcticus 273-4, transcriptional regulator CspA (NC_007204); Psychrobacter cryohalolentis K5, cold-shock DNAbinding domain protein (NC_007969); Psychrobacter sp. B6 CspA-like (EF094463). 


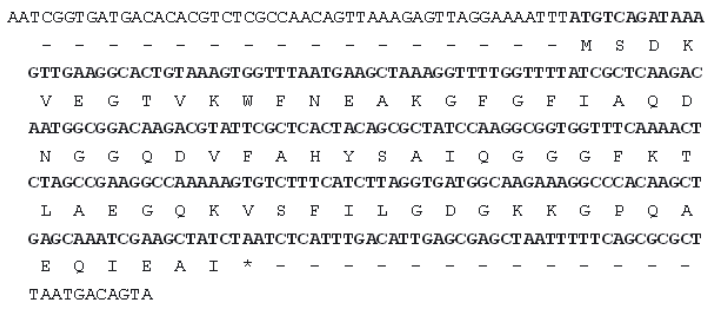

Figure 2. Nucleotide ( $313 \mathrm{bp}$ ) and predicted amino-acid sequences of Psychrobacter sp. B6 cspA region.

The cspA-like coding sequence is shown in bold letters; an asterisk indicates the stop codon.

delin et al., 1994; Feng et al., 1998) and T. maritima (Kremer et al., 2001). The homology model of the CspA-like protein shows that, like other Csps, the Csp molecule from Psychrobacter sp. contains five anti-parallel $\beta$-strands that fold into a $\beta$-barrel built of two $\beta$-sheets. One of these $\beta$-sheets, consisting of strands $\beta_{1}-\beta_{2}-\beta_{3}$, contains two nucleic acid binding motifs on its surface (Fig. 4). The first one, RNP1 - KGFGFI is the same as in the majority of known Csp proteins. The RNP2 motif - VFA/VHY/F is characterized by two changes in amino-acid composition in comparison to the majority of known Csp protein sequences (Fig. 3). Seven aromatic aminoacid residues - W11, F12, F18, F20, F31, Y34 and F42, which are essential for single-stranded nucleic acid binding (Fig. 4) and two lysine residues, K10 and K16, are located on the surface. Thus the 3D structure of the Csp-like protein from Psychrobacter sp. B6 is similar to that of other known Csp molecules. Therefore we assume that Csp B6 plays the role of a molecular chaperone.

\section{DISCUSSION}

CspA-like proteins have been found in cells of many bacterial species but studies on their function and cold shock response focused on mesophilic

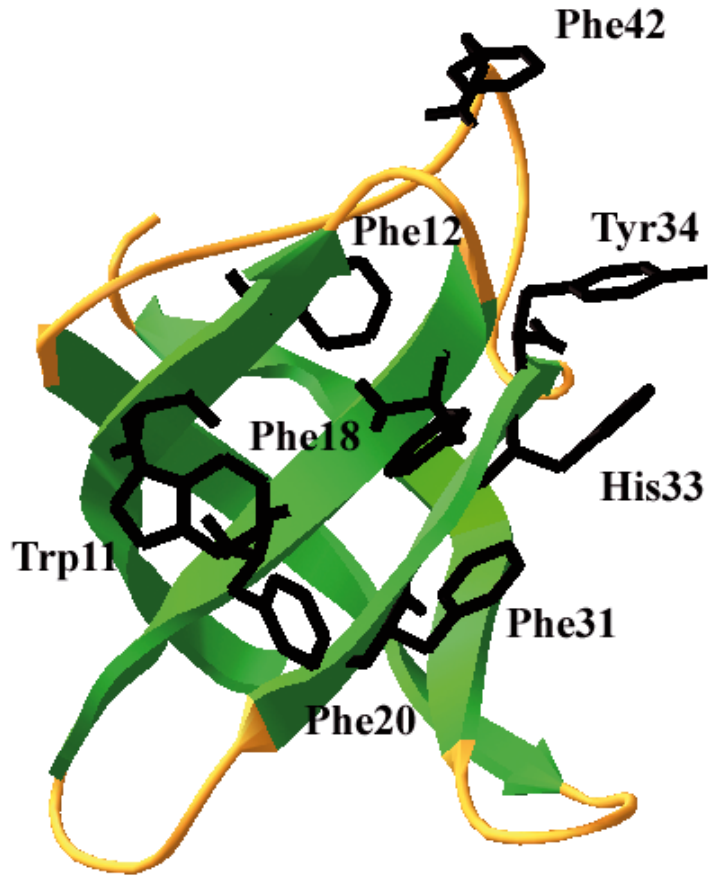

Figure 4. Three-dimensional molecular structure of the CspA-like protein from Psychrobacter sp. B6.

Aromatic residues essential for single stranded nucleic acid binding are highlighted in black.

and thermophilic microorganisms. These proteins have also been detected in psychrophilic and psychrotrophic bacteria, such as Pseudomonas fragi (Hebraud et al., 1994), Arthrobacter globiformis (Berger et al., 1996), Shewanella violacea (Fujii et al., 1999) and strains isolated from polar regions like: Colwellia sp. NJ341 (Wang et al., 2006) and Streptomyces sp. AA8321 (Kim et al., 2007). However, 3D structures of those Csps have not been determined yet.

Sequences of Csps are highly conserved regardless of their source. Because even subtle differences in amino-acid composition and in 3D structure among Csps can lead to differences in their physiological function and interactions with other biomolecules, elucidation of these aspects is necessary.

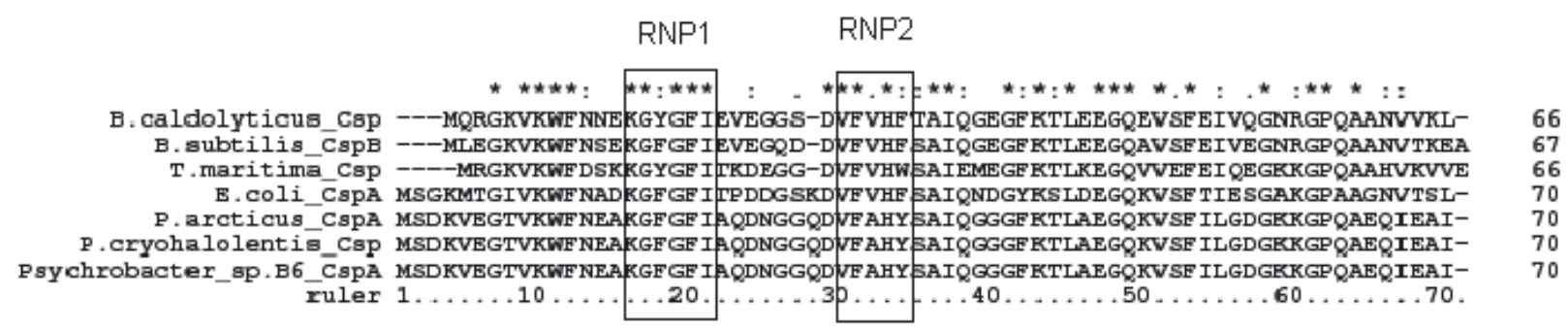

Figure 3. Sequence alignment (CLUSTAL X algorithm) of the CspA-like protein from Psychrobacter sp. B6 with Csp sequences from other bacteria.

The analyzed sequences were obtained from GenBank database: Bacillus caldolyticus Bc-Csp (1C9O_A), Bacillus subtilis CspB (NP_388791), Thermotoga maritima Csp (O54310), Escherichia coli CspA (ACC76580), Psychrobacter arcticus (YP_ 263828), Psychrobacter cryohalolentis Csp (YP_579793). Boxes indicate RNA/ssDNA binding motifs. 
Cloning and sequencing of the $\operatorname{csp} A$-like gene from Antarctic Psychrobacter sp. B6 allowed the modelling of the three-dimensional structure of the protein encoded by this gene. This model provides evidence that the CspA-like protein from Psychrobacter sp. B6 shows high structural similarity to known three-dimensional structures of Csps. The CspA-like molecule consists of two $\beta$-sheets (I: strands $\beta_{1}-\beta_{3}$; II: strands $\beta_{4}$ and $\beta_{5}$ ) folded into a five-stranded $\beta$-barrel structure. Like in the other Csps with known 3D structures, the single-stranded nucleic acid binding motifs, RNP1 and RNP2, are located on the surface of the first $\beta$-sheet. Seven aromatic residues (W11, F12, F18, F20, F31, H33 and Y34) are also exposed on the surface of this $\beta$-sheet while residue F42 is located in the coil region (Fig. 4). Also two lysine residues (K10, K16) with positively charged side chains are located in the same region of the molecule. Aromatic residues, especially those located in RNP1 and RNP2 motifs, are essential for the tight binding of single-stranded nucleic acids (Schröder et al., 1995; Schindler et al., 1998). The other aromatic and nonaromatic side chains are believed to take part in intercalation of the RNA or DNA bases and therefore stabilization of the RNA/DNA-Csp complex.

KGFGFI is the most common sequence of Csps' RNP1 motifs, including the Psychrobacter sp. B6 RNP1 motif. Two phenylalanine aromatic residues of RNP1 are essential for tight binding of RNA/ ssDNA. Substitution at these positions in RNP1 of B. subtilis CspB with non-aromatic residues reduced the binding affinity (Zeeb \& Balbach, 2003). Interestingly, there is one difference in RNP1 motif composition of thermophilic bacteria such as B. caldolyticus (Mueller et al., 2000) and T. maritima (Kremer et al., 2001). They contain Tyr instead of Phe (KGYGFI). Analysis of an Bs-Csp variant with Tyr15 in place of Phe15 revealed a two-fold higher binding affinity as compared to the wild-type CspB (Zeeb \& Balbach, 2003).

The amino-acid composition of the RNP2 nucleic acid binding motif in Psychrobacter sp. B6 CspA-like protein is different from that of the majority of known motifs in mesophilic and thermophilic Csps. For example, the RNP2 motifs of E. coli (Yamanaka et al., 1998), B. subtilis (Schröder et al., 1995) and B. caldolyticus (Mueller et al., 2000) have the sequence VFVHF while in our protein this sequence is VFAHY. It is worth noting that the VFAHY sequence of the RNP2 motif is conserved in the other known Csps from species of Psychrobacter (P. cryohalolentis and $P$. arcticus) and Streptomyces genera, including an Antarctic strain - Streptomyces sp. AA8321 (Kim et al., 2007). For comparison, the RNP2 motif in Csp of the polar psychrophilic bacterium Colwellia sp. NJ341 is VFVHY (Wang et al., 2006). Thus, the occurrence of a tyrosine residue as the last amino acid of this motif may suggest that this feature is common of the Csps from cold adapted bacteria and distinguishes them from their mesophilic counterparts.

The tyrosine residue is replaced by phenylalanine in the RNP2 motif of B. subtilis CspB. Mutation studies have revealed that the highly conserved phenylalanine residues in RNP1 and RNP2 are important for nucleic acid binding and stabilization of this complex (Zeeb \& Balbach, 2003, Zeeb et al., 2006). A Phe $\rightarrow$ Ala substitution in CspB nucleic acid binding motifs reduced the protein binding affinity. Conversely, replacement of Phe30 with Tyr in RNP1 motif of B. subtilis CspB caused a two-fold increase in ssDNA binding affinity in comparison to the wild-type CspB (Zeeb \& Balbach, 2003). In this context, it is possible that the occurrence of Tyr30 (instead of Phe) in RNP2 motifs of CspA-like proteins from cold adapted bacteria can increase their affinity for single-stranded nucleic acids.

In conclusion, the 3D structure of the CspAlike protein from Psychrobacter sp. B6 indicates that it plays a role of a molecular RNA/ssDNA chaperone but this hypothesis needs to be verified experimentally since comprehension of the cold shock response mechanism and cold adaptation of psychrophilic and psychrotrophic microorganisms requires clear defining functions of CspA proteins and regulation of their expression.

\section{Acknowledgements}

We would like to thank Dr. Halina Kalinowska for critical reading and subedit of this manuscript.

\section{REFERENCES}

Bae W, Xia B, Inouye M, Severinov K (2000) Escherichia coli CspA-family RNA chaperones are transcription antiterminators. Proc Natl Acad Sci USA 97: 7784-7789.

Berger F, Morellet N, Menu F, Potier P (1996) Cold shock and cold acclimation proteins in the psychrotrophic bacterium Arthrobacter globiformis SI55. J Bacteriol 178: 2999-3007.

Ermolenko DN, Makhatadze GI (2002) Bacterial cold-shock proteins. Cell Mol Life Sci 59: 1902-1913.

Feng W, Tejero R, Zimmerman DE, Inouye M, Montelione GT (1998) Solution NMR structure and backbone dynamics of the major cold-shock protein (CspA) from Escherichia coli: evidence for conformational dynamics in the single-stranded RNA-binding site. Biochemistry 37: 10881-10896.

Fujii S, Nakasone K, Horikoshi K (1999) Cloning of two cold shock genes, $\operatorname{csp} A$ and $\operatorname{csp} G$, from the deep-sea psychrophilic bacterium Shewanella violacea strain DSS12. FEMS Microbiol Lett 178: 123-128.

Gualerzi CO, Giuliodori AM, Pon CL (2003) Transcriptional and post-transcriptional control of cold-shock genes. J Mol Biol 331: 527-539. 
Hebraud M, Dubole E, Potier P, Labadic J (1994) Effect of growth temperature on the protein levels in psychrotropic bacterium. Pseudomonas fragi. J Bacteriol 176: 4017-4024.

Horn G, Hofweber R, Kremer W, Kalbitzer HR (2007) Structure and function of bacterial cold shock proteins. Cell Mol Life Sci 64: 1457-1470.

Jeffreys AG, Hak KM, SteffanRJ, Foster JW, Bej AK (1998) Growth, survival and characterization of $\operatorname{csp} A$ in Salmonella enteritidis following cold shock. Curr Microbiol 36: 29-35.

Jiang W, Hou Y, Inouye M (1997) CspA, the major coldshock protein of Escherichia coli, is an RNA chaperone. J Biol Chem 272: 196-202.

Jones PG, Inouye M (1994) The cold-shock response - a hot topic. Mol Microbiol 11: 811-818.

Jones PG, Inouye M (1996) RbfA, a 30S ribosomal binding factor, is a cold-shock protein whose absence triggers the cold-shock response. Mol Microbiol 21: 1207-1218.

Jones PG, VanBogelen RA, Neidhardt FC (1987) Induction of proteins in response to low temperature in Escherichia coli. J Bacteriol 169: 2092-2095.

Kim WS, Dunn NW (1997) Identification of cold shock gene in lactic acid bacteria and the effect of cold shock on cryotolerance. Curr Microbiol 35: 59-63.

Kim WS, Khunajakr N, Donn NW (1998) Effect of cold shock on protein synthesis and cryotolerance of cells frozen for long periods in Lactococcus lactis. Cryobiology 37: 86-91.

Kim M-J, Lee YK, Lee HK, Im H (2007) Characterization of cold-shock protein a of antarctic Streptomyces sp. AA8321. Protein J 26: 51-59.

Kloks CP, Spronk CA, Lasonder E, Hoffmann A, Vuister GW, Grzesiek S, Hilbers CW (2002) The solution structure and DNA-binding properties of the cold-shock domain of the human Y-box protein YB-1. J Mol Biol 316: 317-326.

Kloks CP, Tessari M, Vuister GW, Hilbers CW (2004) Cold shock domain of the human Y-box protein YB-1. Backbone dynamics and equilibrium between the native state and a partially unfolded state. Biochemistry 43: 10237-10246.

Kosinski J, Cymerman IA, Feder M, Kurowski MA, Sasin JM, Bujnicki JM (2003) A 'Frankenstein's monster' approach to comparative modelling: merging the finest fragments of Fold-Recognition models and iterative model refinement aided by 3D structure evaluation. Proteins 53 (Suppl 6): 369-379.

Kremer W, Schuler B, Harrieder S, Geyer M, Gronwald W, Welker C, Jaenicke R, Kalbitzer HR (2001) Solution NMR structure of the cold-shock protein from the hyperthermophilic bacterium Thermotoga maritima. Eur J Biochem 268: 2527-2539.

Kurowski MA, Bujnicki JM (2003) GeneSilico protein structure prediction meta-server. Nucleic Acids Res 13: 33053307.

Laskowski RA, MacArthur MW, Moss DS, Thornton JM (1993) PROCHECK - a program to check the stereochemical quality of protein structures. J Appl Cryst 26: 283-291.

Lopez MM, Makhatadze GI (2000) Major cold shock proteins, CspA from Escherichia coli and CspB from Bacillus subtilis, interact differently with single-stranded DNA templates. Biochim Biophys Acta 1479: 196-202.

Luthy R, Bowie JU, Eisenberg D (1992) Assessment of protein models with three-dimensional profiles. Nature 356: 83-85.

Mueller U, Perl D, Schmid F X, Heinemann U (2000) Thermal stability and atomic-resolution crystal structure of the Bacillus caldolyticus cold shock protein. J Mol Biol 297: 975-988.

Nakashima K, Kanamaru K, Mizuno T, Horikoshi K (1996) A novel member of the $\operatorname{csp} A$ family of genes that is induced by cold-shock in Escherichia coli. J Bacteriol 178: 2994-2997.

Newkirk K, Feng W, Jiang W, Tejero R, Emerson SD, Inouye M, Montelione GT (1994) Solution NMR structure of the major cold shock protein (CspA) from Escherichia coli: identification of a binding epitope for DNA. Proc Natl Acad Sci USA 91: 5114-5118.

Peitsch MC (1996) ProMod and Swiss-Model: Internetbased tools for automated comparative protein modelling. Biochem Soc Trans 24: 274-279.

Phadtare S, Inouye M, Severinov K (2002) The nucleic acid melting activity of Escherichia coli CspE is critical for transcription antitermination and cold acclimation of cells. J Biol Chem 277: 7239-7245.

Phadtare S, Hwang J, Severinov K, Inouye M (2003) CspB and CspL, thermostable cold-shock proteins from Thermotoga maritima. Genes Cells 8: 801-810.

Russell NJ, Evans RI, Ter Steeg PF, Hellemons J, Verheul A, Abee T (1995) Membranes as a target for stress adaptation. Int J Food Microbiol 28: 255-261.

Sali A, Blundell TL (1993) Comparative protein modelling by satisfaction of spatial restraints. J Mol Biol 234: 779-815.

Sambrook J, Russell D (2001) Molecular Cloning: A Laboratory Manual. Cold Spring Harbor Laboratory Press.

Sasin JM, Bujnicki JM (2004) COLORADO3D, a web server for the visual analysis of protein structures. Nucleic Acids Res 32 (Web server issue): W586-W589.

Schindelin H, Marahiel MA, Heinemann U (1993) Universal nucleic acid-binding domain revealed by crystal structure of the B. subtilis major cold-shock protein. Nature 364: 164-168.

Schindelin H, Jiang W, Inouye M, Heinemann U (1994) Crystal structure of $\mathrm{CspA}$ the major cold shock protein of Escherichia coli. Proc Natl Acad Sci USA 91: 51195123.

Schindler T, Perl D, Graumann P, Sieber V, Marahiel MA, Schmid FX (1998) Surface-exposed phenylalanines in the RNP1/RNP2 motif stabilize the cold-shock protein CspB from Bacillus subtilis. Proteins 30: 401-406.

Schindler T, Graumann PL, Perl D, Ma S, Schmid FX, Marahiel MA (1999) The family of cold shock proteins of Bacillus subtilis. Stability and dynamics in vitro and in vivo. J Biol Chem 274: 3407-3413.

Schröder K, Graumann P, Schnuchel A, Holak TA, Marahiel MA (1995) Mutational analysis of the putative nucleic acid-binding surface of the cold-shock domain, $\mathrm{CspB}$, revealed an essential role of aromatic and basic residues in binding of single-stranded DNA containing the Y-box motif. Mol Microbiol 27: 699-708.

Schuchel A, Wiltscheck R, Czisch M, Herrel M, Willimsky G, Graumann PL, Marahiel MA, Holak TA (1993) Structure in solution of the major cold-shock protein from Bacillus subtilis. Nature 364: 169-171.

Uppal S, Akkipeddi VS, Jawali N (2008) Posttranscriptional regulation of $\operatorname{csp} E$ in Escherichia coli: involvement of the short 5'-untranslated region. FEMS Microbiol Lett 279: 83-91.

Wang N, Yamanaka K, Inouye M (1999) CspI, the ninth member of the CspA family of Escherichia coli, is induced upon cold shock. J Bacteriol 181: 1603-1609.

Wang Q-F, Miao J-L, Hou Y-H, Ding Y, Li G-Y (2006) Expression of CspA and GST by Antarctic psychrophilic bacterium Colwellia sp. NJ341 at near-freezing temperature. J Microbiol Biotechnol 22: 311-316. 
Willimsky G, Bang H, Fischer G, Marahiel MA (1992) Characterization of $\operatorname{cspB}$, a Bacillus subtilis inducible cold shock gene affecting cell viability at low temperatures. J Bacteriol 174: 6326-6335.

Zeeb M, Balbach J (2003) Single-stranded DNA binding of the cold shock protein CspB from Bacillus subtilis: NMR mapping and mutational characterization. Protein Sci 12: 112-123.
Zeeb M, Max KEA, Weininger U, Löw C, Sticht H, Balbach J (2006) Recognition of T-rich single-stranded DNA by the cold shock protein Bs-CspB in solution. Nucleic Acids Res 34: 4561-4571.

Yamanaka K, Fang L, Inouye M (1998) The CspA family in Escherichia coli: multiple gene duplication for stress adaptation. Mol Microbiol 27: 247-255. 
